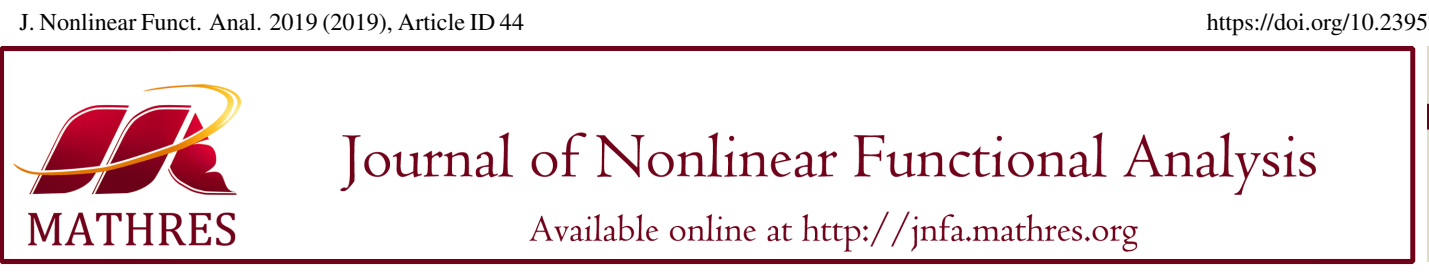

\title{
POPOV SUBGRADIENT EXTRAGRADIENT ALGORITHM FOR PSEUDOMONOTONE EQUILIBRIUM PROBLEMS IN BANACH SPACES
}

\author{
FERDINARD U. OGBUISI ${ }^{1,2}$ \\ ${ }^{1}$ School of Mathematics, Statistics and Computer Science, University of KwaZulu-Natal, Durban, South Africa \\ ${ }^{2}$ Department of Mathematics, University of Nigeria, Nsukka, Nigeria
}

\begin{abstract}
In this paper, we study a modified subgradient extragradient iterative algorithm for the approximation of solutions of pseudomonotone equilibrium problems in the framework of 2-uniformly convex Banach spaces which are also uniformly smooth. Our algorithm is based on two popular iterative methods: the Popov extragradient algorithm and the subgradient extragradient algorithm. We further state and prove a weak convergence result under some mild conditions.
\end{abstract}

Keywords. Popov subgradient extragradient iterative algorithm; Pseudomonotone equilibrium problem; 2-uniformly convex Banach space; Uniformly smooth Banach space.

2010 Mathematics Subject Classification. 47H09, 49J53, 90C25.

\section{INTRODUCTION}

Let $C$ be a nonempty closed and convex subset of a real Banach space $E$. We denote by $E^{*}$ the dual of $E$. Let $f: E \times E \rightarrow \mathbb{R}$ be a bifunction. The Equilibrium Problem (EP) for bifunction $f$ on set $C$ is stated as follows:

$$
\text { Find } x^{*} \in C \text { such that } f\left(x^{*}, y\right) \geq 0, \quad \forall y \in C \text {. }
$$

We denote the solution set of EP (1.1) by $\operatorname{EP}(f, C)$. The EP (1.1) was first introduced by Blum and Oettli [1] and it is well known that EP (1.1) covers many important mathematical models, such as, nonlinear optimization problems, variational inequality problems, nonlinear complementary problems and fixed point problems [1, 2, 3, 4]. The EP (1.1) also generalizes the convex minimization problems which is of great importance in almost all branches of pure and applied sciences because it can be applied in solving many practical problems that arise in areas such as economics, transportation and engineering (see [1,5] and some of the references therein). In recognition of its importance and numerous applications, EP (1.1) have been studied by many authors (see, e.g., $[5,6,7,8,9,10,11,12,13,14,15])$ and many iterative

E-mail address: ferdinard.ogbuisi@unn.edu.ng.

Received June 6, 2019; Accepted October 20, 2019.

(C)2019 Journal of Nonlinear Functional Analysis 
methods, such as, the Proximal Point Method (PPM) [16], auxiliary problem principle method [17] and gap function methods [18] etc, have been developed to solve EP (1.1).

Tran et al. [14] in 2008 introduced the following proximal-like iterative method also referred as the Extragradient Methods (EGM) to solve EP (1.1) in a real Hilbert space under the assumptions that the equilibrium bifunction is pseudomonotone and satisfies the Lipschitz-type condition. For $x_{0} \in C$, let $\left\{x_{n}\right\}$ and $\left\{y_{n}\right\}$ be two sequences generated as follows:

$$
\left\{\begin{array}{l}
y_{n}=\arg \min _{y \in C}\left\{\lambda f\left(x_{n}, y\right)+\frac{1}{2}\left\|x_{n}-y\right\|^{2}\right\}, \\
x_{n+1}=\arg \min _{y \in C}\left\{\lambda f\left(y_{n}, y\right)+\frac{1}{2}\left\|x_{n}-y\right\|^{2}\right\},
\end{array}\right.
$$

where $\lambda>0$ is a suitable parameter. The EGMs have attracted much attention; see, for example, [8, 9, 11, 13, 15, 19]. The advantages of the EGM over the PPM are that it is numerically more easier to compute and it can be used to solve EPs involving a more general class of (pseudomonotone) bifunctions.

The EGM has its own setback as it is required at each step of the iteration to solve two optimization problems on feasible set $C$ and to compute two values of bifunction $f$ at two points $x_{n}$ and $y_{n}$. These can be very costly and can also affect the efficiency of the method if the bifunction and the feasible set have complex structures. To overcome these drawbacks in EGM, Hieu [20] introduced a Modified Extragradient Method (MEGM) in Hilbert spaces for approximating the solution of EP (1.1) involving pseudomonotone bifunctions and obtained a weak convergence result. The feasible set $C$ in the first optimization program in MEGM is replaced by a half space $T_{n}$ and therefore can be solved effectively by using methods of convex quadratic programming ([21], Chapter 8). Also, unlike the EGM, the MEGM only requires to compute a value of the bifunction $f$ at current approximation $y_{n}$.

The purpose of this paper is to introduce an iterative algorithm, which is suitable for finding an element of $E P(f, C)$ for a pseudomonotone bifunction that satisfies the Lipschitz type condition, and obtain a weak convergence result in the framework of 2-uniformly convex Banach spaces which are also uniformly smooth. The results presented in this paper extend the results of Hieu [20] from Hilbert spaces to 2-uniformly convex Banach spaces which are also uniformly smooth.

\section{PRELIMINARIES}

Let $B_{E}=\{x \in E:\|x\|=1\}$. A Banach space $E$ is said to be strictly convex if for any $x, y \in B_{E}$ and $x \neq y$, we have $\frac{\|x+y\|}{2}<1$. $E$ is said to be uniformly convex if for each $\varepsilon \in(0,2]$, there exists $\delta>0$ such that for any $x, y \in B_{E},\|x-y\| \geq \varepsilon$ implies $\frac{\|x+y\|}{2} \leq 1-\delta$. It is known that a uniformly convex Banach space is reflexive and strictly convex. The modulus of convexity of $E$ is the function $\delta_{E}:(0,2] \rightarrow[0,1]$ defined by

$$
\delta_{E}(\varepsilon):=\inf \left\{1-\left\|\frac{x+y}{2}\right\|: x, y \in B_{E} ; \varepsilon=\|x-y\|\right\} .
$$

$E$ is uniformly convex if and only if $\delta_{E}(\varepsilon)>0$ for all $\varepsilon \in(0,2]$ and $p$-uniformly convex if there is a $C_{p}>0$ so that $\delta_{E}(\varepsilon) \geq C_{p} \varepsilon^{p}$ for any $\varepsilon \in(0,2]$. Clearly, every $p$-uniformly convex Banach space is uniformly convex; see, e.g., [22, 23] for more details.

A Banach space $E$ is said to be smooth if the $\operatorname{limit}_{t \rightarrow 0} \frac{\|x+t y\|-\|x\|}{t}$ exists for all $x, y \in B_{E}$. Moreover, $E$ is said to be uniformly smooth if the limit is attained uniformly for $x, y \in B_{E}$. It is well known that Hilbert and the Lebesgue $L_{p}(1<p \leq 2)$ spaces are 2-uniformly convex and uniformly smooth. 
The normalised duality mapping $J: E \rightarrow 2^{E^{*}}$ is defined by

$$
J(x):=\left\{x^{*} \in E^{*}:\left\langle x, x^{*}\right\rangle=\|x\|^{2}=\left\|x^{*}\right\|^{2}\right\}, \forall x \in E .
$$

The normalised duality mapping $J$ possesses the following properties [24]:

(1) If $E$ is a smooth Banach space, then $J$ is single-valued.

(2) If $E$ is a strictly convex Banach space, then $J$ is one-to-one and strictly monotone.

(3) If $E$ is a uniformly smooth Banach space, then $J$ is uniformly norm-to-norm continuous on each bounded subset of $E$.

(4) If $\mathrm{E}$ is a smooth, strictly convex and reflexive Banach space, then $J$ is single-valued, one-to-one and onto.

Let $\mathrm{E}$ be a reflexive, strictly convex and smooth Banach space and let $J$ the normalised duality mapping from $E$ into $E^{*}$. Then $J^{-1}$ is also single-valued, one-to-one, surjective, and is the duality mapping from $E^{*}$ into $E$.

Let $E$ be a smooth Banach space, Alber [25] introduced the following Lyapunov functional

$$
\phi(x, y)=\|x\|^{2}-2\langle x, J y\rangle+\|y\|^{2} .
$$

It can be seen from the definition that $\phi$ satisfies the following conditions.

A1. $(\|x\|-\|y\|)^{2} \leq \phi(x, y) \leq(\|x\|+\|y\|)^{2}$.

A2. $\phi(x, y)=\phi(x, z)+\phi(z, y)+2\langle x-z, J(z)-J(y)\rangle$.

A3. $\phi(x, y)=\langle x, J(x)-J(y)\rangle+\langle y-x, J(y)\rangle \leq\|x|\||J(x)-J(y)\|+\| y-x|\|| y\|$.

If $E$ is a strictly convex and smooth Banach space, then for $x, y \in E, \phi(y, x)=0$ if and only if $x=y$ (see Remark 2.1 in [26]).

Lemma 2.1. [26] Let $E$ be a uniformly convex and smooth Banach space and let $\left\{x_{n}\right\}$ and $\left\{y_{n}\right\}$ be two sequences of $E$. If $\phi\left(x_{n}, y_{n}\right) \rightarrow 0$, and either $\left\{x_{n}\right\}$ or $\left\{y_{n}\right\}$ is bounded, then $x_{n}-y_{n} \rightarrow 0$.

Let $C$ be a nonempty, closed and convex subset of a reflexive, strictly convex and smooth Banach space $E$. Then, for each $x \in E$ (see Alber [25]), there exists a unique element $x_{0} \in C$ (denoted by $\Pi_{C}(x)$ ) such that $\phi\left(x_{0}, x\right)=\min _{y \in C} \phi(y, x)$. The mapping $\Pi_{C}: E \rightarrow C$, defined by $\Pi_{C}(x)=x_{0}$, is called the generalized projection operator from $E$ onto $C$ and $x_{0}$ is called the generalized projection of $x$. In a Hilbert space, $\Pi_{C}=P_{C}$ (the metric projection operator).

Lemma 2.2. [26, 27] Let $C$ be a nonempty closed and convex subset of a smooth Banach space $E$ and $x \in E$. Then, $x_{0}=\Pi_{C}(x)$ if and only if $\left\langle x_{0}-y, J(x)-J\left(x_{0}\right)\right\rangle \geq 0, \forall y \in C$.

Lemma 2.3. [26, 27] Let $E$ be a reflexive, strictly convex and smooth Banach space, let $C$ be a nonempty closed and convex subset of $E$ and let $x \in E$. Then $\phi\left(y, \Pi_{C}(x)\right)+\phi\left(\Pi_{C}(x), x\right) \leq \phi(y, x), \forall y \in C$.

Lemma 2.4. [28] Let $E$ be a 2-uniformly convex and smooth Banach space. Then, for every $x, y \in$ $E, \phi(x, y) \geq c|| x-y \|^{2}$, where $c>0$ is the 2-uniformly convexity constant of $E$.

Next we give an Opial-like inequality for the Lyapunov functional (see [29], Lemma 1). This Opiallike inequality was proved for the more general Bregman distance (see [30], Lemma 5.1 and [31], Lemma 3). For completeness, we state and prove it for our setting here. 
Lemma 2.5. Let $E$ be a smooth Banach space with a weakly sequentially continuous normalised duality mapping $J$ from $E$ into $E^{*}$. Let $\left\{x_{n}\right\}$ be a sequence in $E$ such that $x_{n} \rightarrow u$ weakly for some $u \in E$. Then

$$
\liminf _{n \rightarrow \infty} \phi\left(u, x_{n}\right)<\liminf _{n \rightarrow \infty} \phi\left(v, x_{n}\right) \forall v \in E \text { with } v \neq u \text {. }
$$

Proof.

$$
\begin{aligned}
\phi\left(u, x_{n}\right)-\phi\left(v, x_{n}\right) & =\|u\|^{2}-2\left\langle u, J\left(x_{n}\right)\right\rangle+\left\|x_{n}\right\|^{2}-\left[\|v\|^{2}-2\left\langle v, J\left(x_{n}\right)\right\rangle+\left\|x_{n}\right\|^{2}\right] \\
& =\|u\|^{2}-2\left\langle u-v, J\left(x_{n}\right)\right\rangle-\|v\|^{2} \\
& =\|u\|^{2}-\|v\|^{2}+2\langle v-u, J(u)\rangle-2\langle v-u, J(u)\rangle-2\left\langle u-v, J\left(x_{n}\right)\right\rangle \\
& =-\phi(v, u)+\left\langle u-v, J(u)-J\left(x_{n}\right)\right\rangle .
\end{aligned}
$$

Since $x_{n} \rightarrow u$ weakly and $J$ is weakly sequentially continuous, we have

$$
\lim _{n \rightarrow \infty}\left[\phi\left(u, x_{n}\right)-\phi\left(v, x_{n}\right)\right]=-\phi(v, u) .
$$

Consequently,

$$
\begin{aligned}
\liminf _{n \rightarrow \infty} \phi\left(u, x_{n}\right) & =\liminf _{n \rightarrow \infty}\left[\phi\left(u, x_{n}\right)-\phi\left(v, x_{n}\right)+\phi\left(v, x_{n}\right)\right] \\
& =\lim _{n \rightarrow \infty}\left(\phi\left(u, x_{n}\right)-\phi\left(v, x_{n}\right)\right)+\liminf _{n \rightarrow \infty} \phi\left(v, x_{n}\right) \\
& =-\phi(v, u)+\liminf _{n \rightarrow \infty} \phi\left(v, x_{n}\right) .
\end{aligned}
$$

Since $\phi(v, u)>0$, for $v \neq u$, we obtain

$$
\liminf _{n \rightarrow \infty} \phi\left(u, x_{n}\right)<\liminf _{n \rightarrow \infty} \phi\left(v, x_{n}\right)
$$

The normal cone $N_{C}$ to a set $C$ at a point $x \in C$ is defined by

$$
N_{C}(x):=\left\{x^{*} \in E^{*}:\left\langle x-y, x^{*}\right\rangle \geq 0, \forall y \in C\right\} .
$$

Let $g: C \rightarrow \mathbb{R}$ be a function. The subdifferential of $g$ at $x$ is defined by

$$
\partial g(x)=\left\{w \in E^{*}: g(y)-g(x) \geq\langle w, y-x\rangle, \forall y \in C\right\} .
$$

Lemma 2.6. Let $C$ be a nonempty convex subset of a Banach space $E$ and $g: C \rightarrow \mathbb{R} \cup\{+\infty\}$ be a convex, subdifferentiable and lower semicontinuous function. Furthermore, the function $g$ satisfies the following regularity condition

$$
\text { Either int }(C) \neq \emptyset \text { or } g \text { is continuous at a point in } C \text {. }
$$

Then, $x^{*}$ is a solution to the following convex optimization problem $\min \{g(x): x \in C\}$ if and only if $0 \in \partial g\left(x^{*}\right)+N_{C}\left(x^{*}\right)$, where $\partial g($.$) denotes the subdifferential of g$ and $N_{C}\left(x^{*}\right)$ is the normal cone to $C$ at $x^{*}$. 


\section{Proposed Method}

Let $E$ be a 2-uniformly convex Banach space which is uniformly smooth and let $C$ be a nonempty closed and convex subset of $E$. A bifunction $f: E \times E \rightarrow \mathbb{R}$ is said to be:

i. strongly monotone on $C$ if there exists a constant $\gamma>0$ such that

$$
f(x, y)+f(y, x) \leq-\gamma\|x-y\|^{2}, \forall x, y \in C ;
$$

ii. monotone on $C$ if

$$
f(x, y)+f(y, x) \leq 0, \forall x, y \in C
$$

iii. pseudomonotone on $C$ if

$$
f(x, y) \geq 0 \Rightarrow f(y, x) \leq 0
$$

It is easy to see that $\mathrm{i} \Rightarrow \mathrm{ii} \Rightarrow$ iii. A bifunction $f: E \times E \rightarrow \mathbb{R}$ is said to satisfy Lipschitz-type condition if there exists two constants $L_{1}$ and $L_{2}$ such that

$$
f(x, y)+f(y, z) \geq f(x, z)-L_{1}\|x-y\|^{2}-L_{2}\|y-z\|^{2}, \forall x, y, z \in E .
$$

We now give the following modified subgradient extragradient iterative algorithm for the approximation of solutions of pseudomonotone equilibrium problems in the framework of 2-uniformly convex Banach spaces which are uniformly smooth. First, let us state the following conditions that will be required in the analysis of our convergence result for solving EP (1.1).

\section{Condition B:}

(B1) $f$ is pseudomonotone on $C$ and $f(x, x)=0$, for all $x \in C$;

(B2) $f$ satisfies Lipschitz-type condition on $E$ with Lipschitz-type constants $L_{1}$ and $L_{2}$;

(B3) $f(\cdot, y)$ is sequentially weakly upper semicontinuous on $C$ for each fixed point $y \in C$, i.e., if $\left\{x_{n}\right\} \subset C$ is a sequence converging weakly to $x \in C$, then $\limsup _{n \rightarrow \infty} f\left(x_{n}, y\right) \leq f(x, y)$;

(B4) $f(x, \cdot)$ is convex and subdifferentiable on $E$ for every fixed $x \in C$;

(B5) $0<\lambda<\frac{c}{2 L_{2}+4 L_{1}}$, where $c$ is the 2-uniformly convexity constant of $E$ and $L_{1}$ and $L_{2}$ are the two Lipschitz-type constants of $f$;

(B6) The solution set $E P(f, C)$ of EP (1.1) is nonempty.

It has been proved that under the conditions (B1)-(B4), the solution set $E P(f, C)$ of EP (1.1) is closed and convex [14].

Algorithm 3.1. Initialization: Choose $x_{0} \in E, y_{0} \in C$, a control parameter $\lambda>0$, and compute

Iterative step for $n \geq 1$.

$$
\begin{aligned}
& x_{1}=\arg \min _{y \in C}\left\{\lambda f\left(y_{0}, y\right)+\frac{1}{2} \phi\left(y, x_{0}\right)\right\}, \\
& y_{1}=\arg \min _{y \in C}\left\{\lambda f\left(y_{0}, y\right)+\frac{1}{2} \phi\left(y, x_{1}\right)\right\}
\end{aligned}
$$

Step 1. Select $w_{n} \in \partial_{2} f\left(y_{n-1}, y_{n}\right)=\partial f\left(y_{n-1}, \cdot\right)\left(y_{n}\right)$ and construct a half space

$$
T_{n}=\left\{z \in E:\left\langle J x_{n}-\lambda w_{n}-J y_{n}, z-y_{n}\right\rangle \leq 0\right\} .
$$

Step 2. Solve two strongly convex optimization programs

$$
\left\{\begin{array}{l}
x_{n+1}=\arg \min _{y \in T_{n}}\left\{\lambda f\left(y_{n}, y\right)+\frac{1}{2} \phi\left(y, x_{n}\right)\right\}, \\
y_{n+1}=\arg \min _{y \in C}\left\{\lambda f\left(y_{n}, y\right)+\frac{1}{2} \phi\left(y, x_{n+1}\right)\right\} .
\end{array}\right.
$$


Stopping criterion: If $y_{n+1}=y_{n}=x_{n+1}$, stop. Otherwise, Set $n:=n+1$ and go to Step 1 .

\section{MAin RESUlts}

Now, we are ready to give our main results.

Lemma 4.1. Let $\left\{x_{n}\right\}$ and $\left\{y_{n}\right\}$ be the two sequences generated by Algorithm 3.1.

i. $\lambda\left(f\left(y_{n}, y\right)-f\left(y_{n}, y_{n+1}\right)\right) \geq\left\langle J x_{n+1}-J y_{n+1}, y-y_{n+1}\right\rangle$ for all $y \in C$ and $n \geq 0$.

ii. If $y_{n+1}=y_{n}=x_{n+1}$, then $x_{n+1} \in E P(f, C)$.

iii. For all $x^{*} \in E P(f, C)$, the following estimate holds

$$
\begin{aligned}
\phi\left(x^{*}, x_{n+1}\right) \leq & \phi\left(x^{*}, x_{n}\right)-\left(1-\frac{2 \lambda L_{2}}{c}\right) \phi\left(x_{n+1}, y_{n}\right) \\
& -\left(1-\frac{4 \lambda L_{1}}{c}\right) \phi\left(y_{n}, x_{n}\right)+\frac{4 \lambda L_{1}}{c} \phi\left(x_{n}, y_{n-1}\right) .
\end{aligned}
$$

Proof. i. From the definition of $y_{n+1}$ and Lemma 2.6, we have that

$$
0 \in \partial_{2} \lambda f\left(y_{n}, y_{n+1}\right)+\frac{1}{2} \partial_{1} \phi\left(y_{n+1}, x_{n}\right)+N_{C}\left(y_{n+1}\right) .
$$

Therefore, there exists $w \in \partial_{2} f\left(y_{n}, y_{n+1}\right)$ and $\bar{w} \in N_{C}\left(y_{n+1}\right)$ such that

$$
\lambda w+J y_{n+1}-J x_{n+1}+\bar{w}=0 .
$$

Thus, we have from the definition of $N_{C}$ that

$$
\begin{aligned}
\left\langle J x_{n+1}-J y_{n+1}, y-y_{n+1}\right\rangle & =\lambda\left\langle w, y-y_{n+1}\right\rangle+\left\langle\hat{w}, y-y_{n+1}\right\rangle \\
& \leq \lambda\left\langle w, y-y_{n+1}\right\rangle, \forall y \in C .
\end{aligned}
$$

Again, since $w \in \partial_{2} f\left(y_{n}, y_{n+1}\right)$, we have

$$
\left\langle w, y-y_{n+1}\right\rangle \leq f\left(y_{n}, y\right)-f\left(y_{n}, y_{n+1}\right), \forall y \in C .
$$

Combining (4.2) and (4.3), we obtain

$$
\lambda\left(f\left(y_{n}, y\right)-f\left(y_{n}, y_{n+1}\right)\right) \geq\left\langle J x_{n+1}-J y_{n+1}, y-y_{n+1}\right\rangle, \forall y \in C .
$$

ii. If $y_{n+1}=y_{n}=x_{n+1}$, then from (4.4), Condition (A1) and $\lambda>0$, we obtain $f\left(y_{n}, y\right) \geq 0$, for all $y \in C$. Thus $x_{n+1}=y_{n} \in E P(f, C)$.

iii. From $x_{n+1} \in T_{n}$ and the definition of $T_{n}$, we have

$$
\left\langle J x_{n}-\lambda w_{n}-J y_{n}, x_{n+1}-y_{n}\right\rangle \leq 0 .
$$

Hence

$$
\left\langle J x_{n}-J y_{n}, x_{n+1}-y_{n}\right\rangle \leq \lambda\left\langle w_{n}, x_{n+1}-y_{n}\right\rangle .
$$

Moreover, since $w_{n} \in \partial_{2} f\left(y_{n-1}, y_{n}\right)$, we have

$$
\left\langle w_{n}, y-y_{n}\right\rangle \leq f\left(y_{n-1}, y\right)-f\left(y_{n-1}, y_{n}\right), \forall y \in E
$$

Put $y=x_{n+1}$. From (4.5) and (4.6), we obtain

$$
\left\langle J x_{n}-J y_{n}, x_{n+1}-y_{n}\right\rangle \leq \lambda\left(f\left(y_{n-1}, x_{n+1}\right)-f\left(y_{n-1}, y_{n}\right)\right) .
$$


From the definition of $x_{n+1}$ and by similar arguments as in the proof of (i), we have

$$
\lambda\left(f\left(y_{n}, y\right)-f\left(y_{n}, x_{n+1}\right)\right) \geq\left\langle J x_{n}-J x_{n+1}, y-x_{n+1}\right\rangle, \forall y \in T_{n} .
$$

Now, letting $y=x^{*}$, we have

$$
\lambda\left(f\left(y_{n}, x^{*}\right)-f\left(y_{n}, x_{n+1}\right)\right) \geq\left\langle J x_{n}-J x_{n+1}, x^{*}-x_{n+1}\right\rangle .
$$

Since $x^{*} \in E P(f, C), f\left(x^{*}, y_{n}\right) \geq 0$. Therefore, from the pseudomonotonicity of $f$, we have $f\left(y_{n}, x^{*}\right) \leq 0$. Hence, it follows from relation (4.8) that

$$
-\lambda f\left(y_{n}, x_{n+1}\right) \geq\left\langle J x_{n}-J x_{n+1}, x^{*}-x_{n+1}\right\rangle .
$$

Thus

$$
\left\langle J x_{n}-J x_{n+1}, x_{n+1}-x^{*}\right\rangle \geq \lambda f\left(y_{n}, x_{n+1}\right) .
$$

Since $f$ is Lipschitz-type continuous, we have

$$
\begin{aligned}
f\left(y_{n}, x_{n+1}\right) \geq & \left(f\left(y_{n-1}, x_{n+1}\right)-f\left(y_{n-1}, y_{n}\right)\right) \\
& -L_{1}|| y_{n-1}-y_{n}\left\|^{2}-L_{2}\right\| y_{n}-x_{n+1} \|^{2} .
\end{aligned}
$$

But from the triangular inequality, we have

$$
\begin{aligned}
\left\|y_{n-1}-y_{n}\right\|^{2} & \leq\left(\left\|y_{n-1}-x_{n}\right\|+\left\|x_{n}-y_{n}\right\|\right)^{2} \\
& \leq 2\left(\left\|y_{n-1}-x_{n}\right\|^{2}+\left\|x_{n}-y_{n}\right\|^{2}\right) .
\end{aligned}
$$

We now have from (4.10), (4.11) and Lemma 2.4 that

$$
\begin{aligned}
f\left(y_{n}, x_{n+1}\right) \geq & \left(f\left(y_{n-1}, x_{n+1}\right)-f\left(y_{n-1}, y_{n}\right)\right)-2 L_{1}\left\|y_{n-1}-x_{n}\right\|^{2} \\
& -2 L_{1}\left\|x_{n}-y_{n}\right\|-L_{2}\left\|y_{n}-x_{n+1}\right\|^{2} \\
\geq & \left(f\left(y_{n-1}, x_{n+1}\right)-f\left(y_{n-1}, y_{n}\right)-\frac{2 L_{1}}{c} \phi\left(x_{n}, y_{n-1}\right)\right. \\
& -\frac{2 L_{1}}{c} \phi\left(y_{n}, x_{n}\right)-\frac{L_{2}}{c} \phi\left(x_{n+1}, y_{n}\right) .
\end{aligned}
$$

Therefore, from (4.7), (4.8), (4.9) and (4.12), we have

$$
\begin{aligned}
\left\langle J x_{n}-J x_{n+1}, x_{n+1}-x^{*}\right\rangle \geq & \left\langle J x_{n}-J y_{n}, x_{n+1}-y_{n}\right\rangle-\frac{2 \lambda L_{1}}{c} \phi\left(x_{n}, y_{n-1}\right) \\
& -\frac{2 \lambda L_{1}}{c} \phi\left(y_{n}, x_{n}\right)-\frac{\lambda L_{2}}{c} \phi\left(x_{n+1}, y_{n}\right) .
\end{aligned}
$$

Observe that

$$
\left\langle J x_{n}-J x_{n+1}, x_{n+1}-x^{*}\right\rangle=\frac{1}{2}\left[\phi\left(x^{*}, x_{n}\right)-\phi\left(x^{*}, x_{n+1}\right)-\phi\left(x_{n+1}, x_{n}\right)\right]
$$

and

$$
\begin{aligned}
\left\langle J x_{n}-J y_{n}, x_{n+1}-y_{n}\right\rangle & =-\left\langle J x_{n}-J y_{n}, y_{n}-x_{n+1}\right\rangle \\
& =-\frac{1}{2}\left[\phi\left(x_{n+1}, x_{n}\right)-\phi\left(x_{n+1}, y_{n}\right)-\phi\left(y_{n}, x_{n}\right)\right] \\
& =\frac{1}{2}\left[\phi\left(x_{n+1}, y_{n}\right)+\phi\left(y_{n}, x_{n}\right)-\phi\left(x_{n+1}, x_{n}\right)\right] .
\end{aligned}
$$


Thus, from (4.13), (4.14) and (4.15), we get

$$
\begin{aligned}
\phi\left(x^{*}, x_{n}\right)-\phi\left(x^{*}, x_{n+1}\right) \geq & \phi\left(x_{n+1}, y_{n}\right)+\phi\left(y_{n}, x_{n}\right)-\frac{2 \lambda L_{1}}{c} \phi\left(x_{n}, y_{n-1}\right) \\
& -\frac{2 \lambda L_{1}}{c} \phi\left(y_{n}, x_{n}\right)-\frac{\lambda L_{2}}{c} \phi\left(x_{n+1}, y_{n}\right),
\end{aligned}
$$

which implies

$$
\begin{aligned}
\phi\left(x^{*}, x_{n+1}\right) \leq & \phi\left(x^{*}, x_{n}\right)-\left(1-\frac{2 \lambda L_{2}}{c}\right) \phi\left(x_{n+1}, y_{n}\right) \\
& -\left(1-\frac{4 \lambda L_{1}}{c}\right) \phi\left(y_{n}, x_{n}\right)+\frac{4 \lambda L_{1}}{c} \phi\left(x_{n}, y_{n-1}\right) .
\end{aligned}
$$

Theorem 4.2. Let $C$ be a nonempty closed and convex subset of $E$ and $f: E \times E \rightarrow \mathbb{R}$ be a bifunction such that Conditions (A1)-(A6) are satisfied. Then the sequences $\left\{x_{n}\right\}$ and $\left\{y_{n}\right\}$ generated by Algorithm 3.1 converge weakly to some $p \in E P(f, C)$. Moreover, $p=\lim _{n \rightarrow \infty} \prod_{E P(f, C)}\left(x_{n}\right)$.

Proof. First, we show that $\left\{x_{n}\right\}$ is bounded.

Summing up inequality (4.17) for every $N \geq 1$, we obtain

$$
\begin{aligned}
\phi\left(x^{*}, x_{N+1}\right) \leq & \phi\left(x^{*}, x_{0}\right)+\frac{4 \lambda L_{1}}{c} \phi\left(x_{1}, y_{0}\right) \\
& -\sum_{n=1}^{N}\left(1-\frac{2 \lambda L_{2}}{c}-\frac{4 \lambda L_{1}}{c}\right) \phi\left(x_{n+1}, y_{n}\right) \\
& -\sum_{n=1}^{N}\left(1-\frac{4 \lambda L_{1}}{c}\right) \phi\left(y_{n}, x_{n}\right) .
\end{aligned}
$$

From (B5), we have that

$$
1-\frac{2 \lambda L_{2}}{c}-\frac{4 \lambda L_{1}}{c}>0
$$

and consequently $1-\frac{4 \lambda L_{1}}{c}>0$. This together with inequality (4.18) gives that $\left\{\phi\left(x^{*}, x_{N+1}\right)\right\}$ is bounded for all $N \geq 1$. Thus, $\left\{x_{n}\right\}$ is also bounded. Moreover, it follows from inequality (4.18) that

$$
\sum_{n=1}^{N}\left(1-\frac{2 \lambda L_{2}}{c}-\frac{4 \lambda L_{1}}{c}\right) \phi\left(x_{n+1}, y_{n}\right)<\infty
$$

and

$$
\sum_{n=1}^{N}\left(1-\frac{4 \lambda L_{1}}{c}\right) \phi\left(y_{n}, x_{n}\right)<\infty
$$

Therefore, we have from (B5) that

$$
\lim _{n \rightarrow \infty} \phi\left(x_{n+1}, y_{n}\right)=\lim _{n \rightarrow \infty} \phi\left(y_{n}, x_{n}\right)=0,
$$

which implies that

$$
\lim _{n \rightarrow \infty}\left\|x_{n+1}-y_{n}\right\|=\lim _{n \rightarrow \infty}\left\|y_{n}-x_{n}\right\|=0 .
$$

Now from the triangular inequality, we have

$$
\left\|x_{n+1}-x_{n}\right\| \leq\left\|x_{n+1}-y_{n}\right\|+\left\|y_{n}-x_{n}\right\| \rightarrow 0, n \rightarrow \infty .
$$


Also,

$$
|| y_{n-1}-y_{n}|| \leq\left\|y_{n-1}-x_{n}||+|| x_{n}-y_{n}\right\| \rightarrow 0, n \rightarrow \infty
$$

Since $\left\{x_{n}\right\}$ is bounded, there exists a subsequence $\left\{x_{n_{i}}\right\}$ of $\left\{x_{n}\right\}$ such that $x_{n_{i}} \rightarrow p$. Also, since $\| x_{n_{i}}-$ $y_{n_{i}}|| \rightarrow 0$, we have $y_{n_{i}} \rightarrow p$. Since $C$ is a closed and convex subset of $E$, we have that $C$ is weakly closed. Hence, it follows from $\left\{y_{n_{i}}\right\} \subset C$ that $p \in C$. Also, from (4.4), with $n=n_{i}$ and the Lipschitz-type continuity of $f$, we have

$$
\begin{aligned}
\lambda f\left(y_{n_{i}}, y\right) \geq & \lambda f\left(y_{n_{i}}, y_{n_{i}+1}\right)+\left\langle J x_{n_{i}+1}-J y_{n_{i}+1}, y-y_{n_{i}+1}\right\rangle \\
\geq & \lambda\left(f\left(y_{n_{i}-1}, y_{n_{i}+1}\right)-f\left(y_{n_{i}-1}, y_{n_{i}}\right)\right)-\lambda L_{1}\left\|y_{n_{i}-1}-y_{n_{i}}\right\|^{2} \\
& -\lambda L_{2}|| y_{n_{i}}-y_{n_{i}+1} \|^{2}+\left\langle J x_{n_{i}+1}-J y_{n_{i}+1}, y-y_{n_{i}+1}\right\rangle .
\end{aligned}
$$

Again from (4.4) with $n=n_{i}-1$, we obtain

$$
\lambda\left(f\left(y_{n_{i}-1}, y\right)-f\left(y_{n_{i}-1}, y_{n_{i}}\right) \geq\left\langle J x_{n_{i}}-J y_{n_{i}}, y-y_{n_{i}}\right\rangle, \forall y \in C .\right.
$$

This with $y=y_{n_{i}+1}$ leads to

$$
\lambda\left(f\left(y_{n_{i}-1}, y\right)-f\left(y_{n_{i}-1}, y_{n_{i}}\right) \geq\left\langle J x_{n_{i}}-J y_{n_{i}}, y_{n_{i}+1}-y_{n_{i}}\right\rangle .\right.
$$

Combining relations (4.24) and (4.25), we have

$$
\begin{aligned}
\lambda f\left(y_{n_{i}}, y\right) \geq & \left\langle J x_{n_{i}}-J y_{n_{i}}, y_{n_{i}+1}-y_{n_{i}}\right\rangle \\
& -\lambda L_{1}|| y_{n_{i}-1}-y_{n_{i}}\left\|^{2}-\lambda L_{2}\right\| y_{n_{i}}-y_{n_{i}+1} \|^{2} \\
& +\left\langle J x_{n_{i}+1}-J y_{n_{i}+1}, y-y_{n_{i}+1}\right\rangle, \forall y \in C .
\end{aligned}
$$

Passing to the limit in (4.26) as $n_{i} \rightarrow \infty$ and using hypothesis (B3), (4.21) and the uniformly norm-tonorm continuity of the normalised duality mapping $\mathrm{J}$, we have $f(p, y) \geq 0$ for all $y \in C$ or $p \in E P(f, C)$.

Next, we show that $x_{n} \rightarrow p$. Suppose for contradiction that there is a subsequence $\left\{x_{n_{j}}\right\}$ of $\left\{x_{n}\right\}$ such that $x_{n_{j}} \rightarrow q$ and $q \neq p$. From (4.1) and $1-\frac{2 \lambda L_{2}}{c}-\frac{4 \lambda L_{1}}{c}>0$, we have

$$
\phi\left(x^{*}, x_{n+1}\right)+\frac{4 \lambda L_{1}}{c} \phi\left(x_{n+1}, y_{n}\right) \leq \phi\left(x^{*}, x_{n}\right)+\frac{4 \lambda L_{1}}{c} \phi\left(x_{n}, y_{n-1}\right) .
$$

Therefore, for all $x^{*} \in E P(f, C)$, there is

$$
\lim _{n \rightarrow \infty}\left(\phi\left(x^{*}, x_{n}\right)+\frac{4 \lambda L_{1}}{c} \phi\left(x_{n}, y_{n-1}\right)\right) \in \mathbb{R}
$$


Applying Lemma 2.5 twice, we obtain

$$
\begin{aligned}
\lim _{n \rightarrow \infty}\left(\phi\left(p, x_{n}\right)+\frac{4 \lambda L_{1}}{c} \phi\left(x_{n}, y_{n-1}\right)\right) & =\lim _{i \rightarrow \infty}\left(\phi\left(p, x_{n_{i}}\right)+\frac{4 \lambda L_{1}}{c} \phi\left(x_{n_{i}}, y_{n_{i}-1}\right)\right) \\
& =\liminf _{i \rightarrow \infty} \phi\left(p, x_{n_{i}}\right)<\liminf _{i \rightarrow \infty} \phi\left(q, x_{n_{i}}\right) \\
& =\lim _{i \rightarrow \infty}\left(\phi\left(q, x_{n_{i}}\right)+\frac{4 \lambda L_{1}}{c} \phi\left(x_{n_{i}}, y_{n_{i}-1}\right)\right) \\
& =\lim _{n \rightarrow \infty}\left(\phi\left(q, x_{n}\right)+\frac{4 \lambda L_{1}}{c} \phi\left(x_{n}, y_{n-1}\right)\right) \\
& =\lim _{j \rightarrow \infty}\left(\phi\left(q, x_{n_{j}}\right)+\frac{4 \lambda L_{1}}{c} \phi\left(x_{n_{j}}, y_{n_{j}-1}\right)\right) \\
& =\liminf _{j \rightarrow \infty}\left(\phi\left(q, x_{n_{j}}\right)<\liminf _{j \rightarrow \infty} \phi\left(p, x_{n_{j}}\right)\right. \\
& =\lim _{j \rightarrow \infty}\left(\phi\left(p, x_{n_{j}}\right)+\frac{4 \lambda L_{1}}{c} \phi\left(x_{n_{j}}, y_{n_{j}-1}\right)\right) \\
& =\lim _{n \rightarrow \infty}\left(\phi\left(p, x_{n}\right)+\frac{4 \lambda L_{1}}{c} \phi\left(x_{n}, y_{n-1}\right)\right) .
\end{aligned}
$$

This is an absurdity. Thus, we conclude that $p=q$.

We proceed to show that

$$
p=\lim _{n \rightarrow \infty} \Pi_{E P(f, C)} x_{n} .
$$

Clearly,

$$
\begin{aligned}
\phi\left(\Pi_{E P(f, C)} x_{n+1}, x_{n+1}\right) & \leq \phi\left(\Pi_{E P(f, C)} x_{n}, x_{n+1}\right) \\
& \leq \phi\left(\Pi_{E P(f, C)} x_{n}, x_{n}\right)+\frac{4 \lambda L_{1}}{c} \phi\left(x_{n}, y_{n-1}\right) .
\end{aligned}
$$

Since $\sum_{n=1}^{\infty} \phi\left(x_{n}, y_{n-1}\right)<\infty$, we have

$$
\lim _{n \rightarrow \infty} \phi\left(\Pi_{E P(f, C)} x_{n}, x_{n}\right) \in \mathbb{R} .
$$

Thus it follows from Lemma 2.3 that

$$
\begin{aligned}
\phi\left(\Pi_{E P(f, C)} x_{n}, \Pi_{E P(f, C)} x_{m}\right) \leq & \phi\left(\Pi_{E P(f, C)} x_{n}, x_{m}\right)-\phi\left(\Pi_{E P(f, C)} x_{m}, x_{m}\right) \\
\leq & \phi\left(\Pi_{E P(f, C)} x_{n}, x_{m-1}\right)-\phi\left(\Pi_{E P(f, C)} x_{m}, x_{m}\right) \\
& +\frac{4 \lambda L_{1}}{c} \phi\left(x_{m-1}, y_{m-2}\right) \\
& \vdots \\
\leq & \phi\left(\Pi_{E P(f, C)} x_{n}, x_{n}\right)-\phi\left(\Pi_{E P(f, C)} x_{m}, x_{m}\right) \\
& +\frac{4 \lambda L_{1}}{c} \sum_{k=n}^{m} \phi\left(x_{k-1}, y_{k-2}\right), m>n .
\end{aligned}
$$

Passing to the limit in (4.29) as $m, n \rightarrow \infty$, we obtain

$$
\lim _{n, m \rightarrow \infty} \phi\left(\Pi_{E P(f, C)} x_{n}, \Pi_{E P(f, C)} x_{m}\right)=0,
$$

which implies that

$$
\lim _{n, m \rightarrow \infty}\left\|\Pi_{E P(f, C)} x_{n}-\Pi_{E P(f, C)} x_{m}\right\|=0 .
$$


Thus $\left\{\Pi_{E P(f, C)} x_{n}\right\}$ is a Cauchy sequence. Hence there exists the limit

$$
\lim _{n \rightarrow \infty} \Pi_{E P(f, C)} x_{n}=z \in E P(f, C) .
$$

From Lemma 2.2, we have

$$
\left\langle J \Pi_{E P(f, C)} x_{n}-J x_{n}, p-\Pi_{E P(f, C)} x_{n}\right\rangle \geq 0 .
$$

Passing to the limit as $n \rightarrow \infty$, we obtain $\langle J z-J p, p-z\rangle \geq 0$, which implies

$$
\phi(z, p)+\phi(p, z)=2\langle J z-J p, z-p\rangle \leq 0 .
$$

Hence $z=p$.

\section{Applications}

Let $E$ be a real Banach space and let $C$ be a nonempty, closed and convex subset of $E$. Let $A: E \rightarrow E^{*}$ be an operator. The variational inequality problem, (for short, $\operatorname{VI}(A, C)$ ) associated with $A$ and $C$ is defined as: find $x \in C$ such that

$$
\langle A x ; z-x\rangle \geq 0 ; \forall z \in C .
$$

We denote by $\Gamma$ the solution set of variational inequality (5.1).

Remark 5.1. In the special case where $f(x, y)=\langle A x, y-x\rangle$, the EP (1.1) becomes the $V I(A, C)(5.1)$. Moreover Algorithm 3.1 is reduced to the following.

Algorithm 5.2. Initialization: Choose $x_{0} \in E, y_{0} \in C$, a control parameter $\lambda>0$, and compute

$$
\begin{aligned}
& x_{1}=\Pi_{C} J^{-1}\left(J x_{0}-\lambda A y_{0}\right), \\
& y_{1}=\Pi_{C} J^{-1}\left(J x_{1}-\lambda A y_{0}\right) .
\end{aligned}
$$

Iterative step for $n \geq 1$.

Step 1. construct a half space

$$
T_{n}=\left\{z \in E:\left\langle J x_{n}-\lambda A y_{n-1}-J y_{n}, z-y_{n}\right\rangle \leq 0\right\} .
$$

Step 2.

$$
\left\{\begin{array}{l}
x_{n+1}=\Pi_{T_{n}} J^{-1}\left(J x_{n}-\lambda y_{n}\right), \\
y_{n+1}=\Pi_{C} J^{-1}\left(J x_{n+1}-\lambda A y_{n}\right) .
\end{array}\right.
$$

Stopping criterion: If $y_{n+1}=y_{n}=x_{n+1}$, stop. Otherwise, Set $n:=n+1$ and go to Step 1 .

Therefore, if we let $f(x, y)=\langle A x, y-x\rangle$, and assume that $A: E \rightarrow E^{*}$ satisfies the following conditions:

(1) $A$ is a pseudo-monotone operator on $C$, that is, for all $x, y \in C,\langle A x, y-x\rangle \geq 0 \Rightarrow\langle A y, x-y\rangle \leq 0$;

(2) $A$ is L-Lipschitz-continuous on $E$, that is, there exists a constant $L>0$ such that $\|A x-A y\| \leq L\|x-y\|$ for all $x, y \in E$ (It was shown [11] that if $A$ is a Lipschitz continuous operator, $f(x, y)=\langle A x, y-x\rangle\rangle$ satisfies the Lipschitz-type condition with $L_{1}=L_{2}=\frac{L}{2}$.

(3) $A$ is sequentially weakly continuous on $C$, that is, for each sequence $\left\{x_{n}\right\} \subset C$, we have that $\left\{x_{n}\right\}$ converges weakly to $x \in E$ implies $\left\{A x_{n}\right\}$ converges weakly to $A x$. 
(4) The solution set of $\operatorname{VI}(A, C)(5.1)$ is nonempty.

Then from Lemma 4.1 and Theorem 4.2 with $f(x, y)=\langle A x, y-x\rangle$, and $A$ satisfying conditions (1)-(4), we obtain the desired convergence results.

\section{Acknowledgements}

The work was based on the research wholly supported by the National Research Foundation (NRF) of South Africa (Grant NO. 111992). Opinions expressed and conclusions arrived are those of the author and are not necessarily to be attributed to the NRF.

\section{REFERENCES}

[1] E. Blum, W. Oettli, From optimization and variational inequality to equilibrium problems, Math. Stud. 63 (1994), 127-149.

[2] I. Konnov, Combined Relaxation Methods for Variational Inequalities, Lecture Notes in Economics and Mathematical Systems, 495, Springer-Verlag, Berlin, 2001.

[3] I. Konnov, Equilibrium Models and Variational Inequalities, Mathematics in Science and Engineering, 210, Elsevier B. V., Amsterdam, 2007.

[4] L.D. Muu, W. Oettli, Convergence of an adaptive penalty scheme for finding constrained equilibria, Nonlinear Anal. 18 (1992), 1159-1166.

[5] L.Q. Anh, T. Bantaojai, N.P. Duc, T.Q. Duy, R. Wangkeeree, Convergence of solutions to lexicographic equilibrium problems, J. Appl. Numer. Optim. 1 (2019), 39-51.

[6] P.L. Combettesm, S. A. Hirstoaga, Equilibrium programming in Hilbert spaces, J. Nonlinear Convex Anal. 6 (2005), 117-136.

[7] X. Qin, A. Petrusel, J.C. Yao, CQ iterative algorithms for fixed points of nonexpansive mappings and split feasibility problems in Hilbert spaces, J. Nonlinear Convex Anal. 19 (2018), 157-165.

[8] X. Qin, J.C. Yao, Projection splitting algorithms for nonself operators, J. Nonlinear Convex Anal. 18 (2017), 925-935.

[9] S.Y. Cho, B.A. Bin Dehaish, X. Qin, Weak convergence of a splitting algorithm in Hilbert spaces, J. Appl. Anal. Comput. 7 (2017), 427-438.

[10] D.V. Hieu, P.K. Anh, L.D. Muu, Modified hybrid projection methods for finding common solutions to variational inequality problems, Comput. Optim. Appl. 66 (2017), 75-96.

[11] D.V. Hieu, L.D. Muu, P.K. Anh, Parallel hybrid extragradient methods for pseu- domonotone equilibrium problems and nonexpansive mapping, Numer. Algo. 73 (2016), 197-217.

[12] A.N. Iusem, G. Kassay, W. Sosa, On certain conditions for the existence of solutions of equilibrium problems, Math. Program. 116 (2009), 259-273.

[13] T.T.V. Nguyen, J.J. Strodiot, V.H. Nguyen, Hybrid methods for solving simultaneously an equilibrium problem and countably many fixed point problems in a Hilbert space, J. Optim. Theory Appl. 160 (2014), 809-831.

[14] D.Q. Tran, M.L. Dung, V.H. Nguyen, Extragradient algorithms extended to equilibrium problems, Optimization, 57 (2008), 749-776.

[15] P.T. Vuong, J.J. Strodiot, V.H. Nguyen, On extragradient-viscosity methods for solving equilibrium and fixed point problems in a Hilbert space, Optimization, 64 (2015), 429-451.

[16] A. Moudafi, Proximal point algorithm extended to equilibrium problems, J. Nat. Geom. 15 (1999), 91-100.

[17] G. Mastroeni, On auxiliary principle for equilibrium problems, Publicatione del Dipartimento di Mathematica dell, Universita di Pisa, 3 (2000), 1244-1258.

[18] G. Mastroeni, Gap functions for equilibrium problems, J. Global Optim. 27 (2003), 411-426.

[19] Y. Censor, A. Gibali, S. Reich, Strong convergence of subgradient extragradient methods for the variational inequality problem in Hilbert space, Optim. Methods Softw. 26 (2011), 827-845.

[20] D.V. Hieu, Modified subgradient extragradient algorithm for pseudomonotone equilibrium problems, Bull. Korean Math. Soc. 55 (2018), 1503-1521. 
[21] S. Boyd, L. Vandenberghe, Convex Optimization, Cambridge University Press, Cambridge, 2004.

[22] K. Ball, E.A. Carlen, E.H. Lieb, Sharp uniform convexity and smoothness inequalities for trace norms, Invent. Math. 115 (1994), 463-482.

[23] Y. Takahashi, K. Hashimoto, M. Kato, On sharp uniform convexity, smoothness, and strong type, cotype inequalities, J. Nonlinear Convex Anal. 3 (2002), 267-281.

[24] Y.I.Alber, S.Reich, An iterative method for solving a class of nonlinear operator equations in Banach spaces, PanAmer. Math. J. 4 (1994), 39-54.

[25] Y.I. Alber, Metric and generalized projection operators in Banach spaces: Properties and applications, in: A.G. Kartsatos (Ed.), Theory and Applications of Nonlinear Operators of Accretive and Monotone Type, in: Lecture Notes Pure Appl. Math., vol. 178, Dekker, New York, 1996, pp. 15-50.

[26] S. Matsushita, W. Takahashi, A strong convergence theorem for relatively nonexpansive mappings in a Banach space, J. Approx. Theory 134 (2005), 257-266.

[27] H. Iiduka, W. Takahashi, Weak convergence of a projection algorithm for variational inequalities in a Banach space, J. Math. Anal. Appl. 339 (2008), 668-679.

[28] K. Nakajo, Strong convergence for gradient projection method and relatively nonexpansive mappings in Banach spaces, Appl. Math. Comput. 271 (2015), 251-258.

[29] Z. Opial, Weak convergence of the sequence of successive approximations for nonexpansive mappings, Bull. Amer. Math. Soc. 73 (1967), 591-597.

[30] Y-Y. Huang, J-C. Jeng, T-Y. Kuo, C-C. Hong, Fixed point and weak convergence theorems for point-dependent $\lambda$-hybrid mappings in Banach spaces, Fixed Point Theory Appl. 2011 (2011), Article ID 105.

[31] C.-T, Pang, E. Naraghirad, C.-F. Wen, Weak convergence theorems for Bregman relatively nonexpansive mappings in Banach spaces, J. Appl. Math. 2014 (2014), Art. ID 573075. 